\title{
Roughness and stability of silicon on insulator surfaces
}

M. Czubanowski, C. Tegenkamp, W. Ernst, and H. Pfnür

Citation: Appl. Phys. Lett. 84, 350 (2004);

View online: https://doi.org/10.1063/1.1641181

View Table of Contents: http://aip.scitation.org/toc/apl/84/3

Published by the American Institute of Physics

\section{Articles you may be interested in}

Anomalous molecular orbital variation upon adsorption on a wide band gap insulator

The Journal of Chemical Physics 132, 214706 (2010); 10.1063/1.3431755

Correlation of electronic and local structure of 4-hydroxy-thiophenol on $\mathrm{NaCl}(100)$ and $\mathrm{Ag}(100)$

The Journal of Chemical Physics 118, 7578 (2003); 10.1063/1.1562193

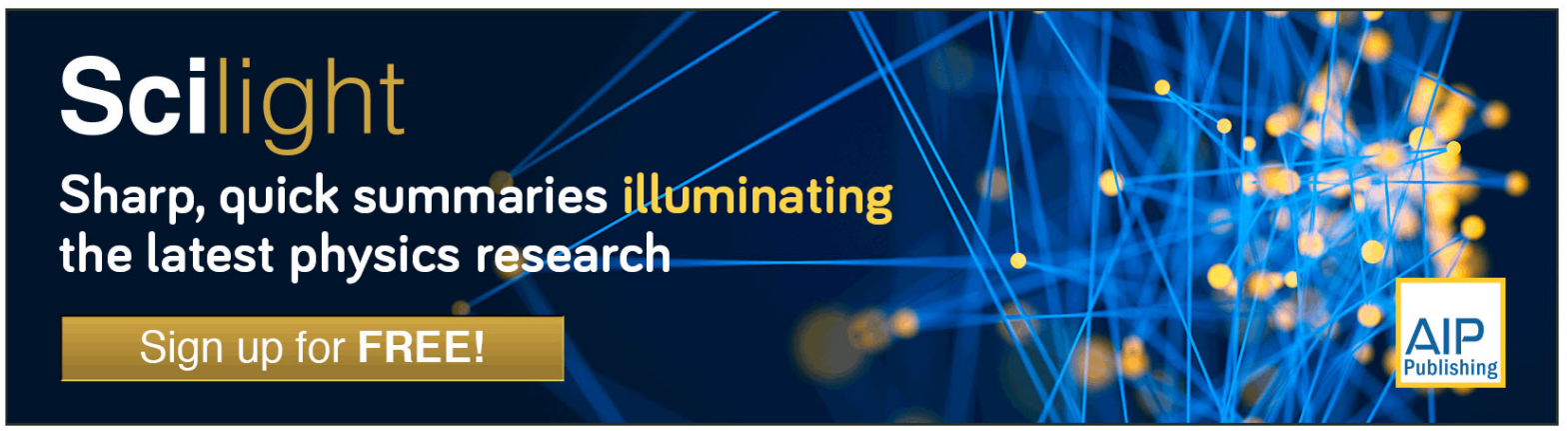




\title{
Roughness and stability of silicon on insulator surfaces
}

\author{
M. Czubanowski, C. Tegenkamp, ${ }^{\text {a) }}$ W. Ernst, and H. Pfnür \\ Institut für Festkörperphysik, Universität Hannover, Appelstraße 2, D-30167 Hannover, Germany
}

(Received 13 August 2003; accepted 19 November 2003)

\begin{abstract}
The feasibility of low temperature processes (below $800^{\circ} \mathrm{C}$ ) to obtain in situ atomically clean and smooth surfaces on (100) oriented silicon on insulator material (SOI) with negligible variation of the top Si film thickness was tested. These steps were characterized using low-energy electron diffraction and atomic force microscopy supplemented by conductivity measurements. The most promising method for obtaining atomically smooth and continuous SOI films is the evaporation of $\mathrm{Si}$ at $750^{\circ} \mathrm{C}$ at a flux of $0.15 \mathrm{ML} / \mathrm{min}$. For lower rates [113]-oriented pits are formed within the SOI layer. It turned out that mobile and volatile oxide formation at the $\mathrm{Si} / \mathrm{SiO}_{2}$ interface in these materials can occur already at temperatures below $1000^{\circ} \mathrm{C}$, leading to the destruction of the buried oxide layer. (C) 2004 American Institute of Physics. [DOI: 10.1063/1.1641181]
\end{abstract}

The ongoing miniaturization of electronic devices not only makes the structures in electronic circuits smaller and smaller, it also has increased the influence of the properties of interfaces more and more. It has reached a level that enforces the geometric, chemical, and electronic definition and control of surfaces and interfaces on the atomic scale.

Most circuits today are based on Si technology. The use of silicon-on-insulator (SOI) material has become attractive in recent years, since a thin single crystalline film of $\mathrm{Si}$ is directly bonded to insulating $\mathrm{SiO}_{2}$. It not only facilitates technology by reducing the number of necessary steps of processing, it also allows minimization of parasitic capacitance effects. From a physical point of view, ultrathin SOI surfaces are interesting because of their electronic structure. As investigated in detail with scanning tunneling microscopy, ${ }^{1}$ already the lowest concentrations of missing surface $\mathrm{Si}$ atoms form characteristic metallic defect states, which lead to pinning of the Fermi level, i.e., for $p$-doped silicon $(\rho \approx 10 \Omega \mathrm{cm})$ a Schottky barrier at the vacuum interface occurs. Therefore, thin topmost Si films $(<100 \mathrm{~nm}$, depending on the level of doping) are charge depleted.

As a general rule, a heterostructure like SOI with atomically sharp interfaces is metastable, both for entropical and for energetic reasons. Therefore, it is susceptible to high temperature treatment. As a consequence, it is difficult or even impossible to use the standard procedures in UHV for in situ removal of oxides and other contaminations by heating samples above $1000^{\circ} \mathrm{C}$. However, steps like these are necessary to obtain atomically smooth and clean $\mathrm{Si}$ surfaces of single crystalline $\mathrm{Si}$, since successful processing of surfaces, e.g., in molecular beam epitaxy, depends on roughness on the atomic scale and on traces of contaminations. Therefore, $e x$ situ cleaning steps are generally not sufficient. Inevitably, a process that desorbs $\mathrm{SiO}_{2}$ at the vacuum interface must also make this species mobile at the inner interfaces, thus deteriorating or even destroying them. ${ }^{2,3}$ Therefore, alternatives have to be tested.

A further problem seems to be the different degree of metastability from the various production schemes of SOI.

${ }^{a}$ Electronic mail: tegenkamp@ fkp.uni-hannover.de
Whereas the separation by implantated oxygen (SIMOX) technology forms the SOI structure by implantaion of highenergetic oxygen ions, the UNIBOND ${ }^{\circledR}$ flow process takes advantage of the special bonding of two adequately prepared Si wafers. Prior to bonding, both wafers are thermally oxidized. As judged from the published literature, ${ }^{1,4}$ the interfaces obtained with the former process seem to be more stable.

In view of these problems, we tried to optimize in situ preparation methods for atomically smooth, contiguous, and clean Si films on SOI under UHV conditions and at temperatures below $800^{\circ} \mathrm{C}$. Characterization of surface morphology and of surface chemistry has been carried out using a combination of low-energy electron diffraction (LEED), Auger electron spectroscopy (AES), and ex situ atomic force microscopy (AFM). We show that the removal of $\mathrm{SiO}_{2}$ by evaporation of silicon at elevated substrate temperatures ${ }^{5-7}$ is a viable method also for extremely thin SOI films. It can be carried out at temperatures as low as $750^{\circ} \mathrm{C}$ and at extremely small evaporation rates without producing etch pits. Thus, the thickness of the topmost $\mathrm{Si}$ layers changes only very little. As a second method, a combination of sputtering with Ar ions, annealing, and smoothing the surface by adsorption of molecular oxygen has been performed, which seems to be an alternative to the former method. Finally, the instability of the insulating film of the sandwich structure is demonstrated by high temperature treatments. For our samples produced with the UNIBOND® process we observed thermal stability of the buried oxide layer (BOX) only for substrate temperatures lower than $900{ }^{\circ} \mathrm{C}$. This finding will be discussed in terms of suboxides $\left(\mathrm{SiO}_{x}, 0<x<2\right)$ located at the $\mathrm{Si} / \mathrm{SiO}_{2}$ interface.

Our SOI samples had a 200-nm-thick Si film on top of the 400-nm-thick BOX film. Macroscopic conductivity measurements have been carried out in situ both along the SOI film and between film and bulk $\mathrm{Si}$ in order to check qualitatively the insulating properties of the BOX. Different preparation techniques have been tested using always SOI samples from the same wafer. After a high temperature treatment they were replaced in order to make the indiviual steps comparable. 


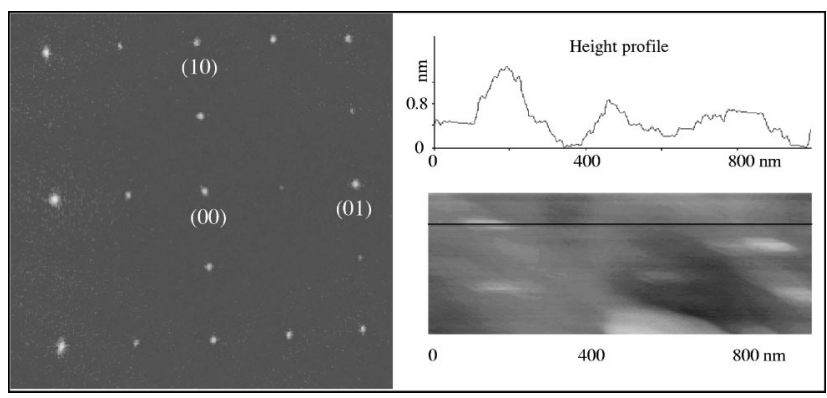

FIG. 1. LEED pattern (left) and AFM image (right, together with a height profile) of a SOI surface cleaned by $\mathrm{Si}$ evaporation at $T=750{ }^{\circ} \mathrm{C}$. Evaporation rate was $0.15 \mathrm{ML} / \mathrm{min}$. The electron energy was $111 \mathrm{eV}$.

All manipulation steps of the SOI sample like evaporation, sputtering, heating, etc., were performed in an UHV chamber at a base pressure of $10^{-8} \mathrm{~Pa}$. The UHV system was equipped with LEED, AES, and a quadrupole mass analyzer (QMA). Silicon was evaporated out of a graphite crucible heated by electron bombardment. The evaporation rate was monitored with a quarz microbalance. It was calibrated by calculating the change of frequency of the quarz microbalance with increasing film thickness assuming an ideal layer-by-layer growth. The cleanness of silicon and also of oxygen and argon for the purpose of smoothing and sputtering was checked by AES and with the QMA, respectively. The SOI sample was heated by direct current through the bulk material and the temperature was controlled by an infrared pyrometer. The sample temperature was carefully calibrated in advance using a $\mathrm{Ni} / \mathrm{NiCr}$ thermocouple, which was directly attached to a dummy Si sample of the same size in place of the SOI samples on the sample holder. Thus, the uncertainty of the temperature reading could be reduced to $\pm 5^{\circ} \mathrm{C}$. The AFM (Park M5) and additional conductivity measurements have been done ex situ.

In a first step, we tried to remove the thin cap oxide, grown under ambient conditions, by evaporation of $\mathrm{Si}$ at temperatures below $800^{\circ} \mathrm{C}$ using the reaction: $\mathrm{SiO}_{2}(\mathrm{ad})$ $+\mathrm{Si}(\mathrm{ad}) \rightarrow \mathrm{SiO}$ (gas), at a temperature and at evaporation rates as low as possible. According to a generally accepted model, ${ }^{8}$ this process at the surface competes with the formation of $\mathrm{Si}$ monomers at the $\mathrm{SiO}_{2} / \mathrm{Si}$ interface, which may be produced by $\mathrm{Si}$ diffusing through the oxide. This leads to an etching process also at the inner interface and formation of etch pits ${ }^{5,9}$ with (111)- or (113)-oriented facets that turn out to be the most stable facets under this reaction. A smooth surface bare of oxygen can therefore only be achieved when the reaction of evaporated $\mathrm{Si}$ with $\mathrm{SiO}_{2}$ at the vacuum interface is much faster than the reaction at the inner surface. This depends on evaporation rate and temperature, which controls diffusivities and rates of desorption. However, the Si evaporation rate of $1-1.5 \AA / s$ at a temperature of $780^{\circ} \mathrm{C}$ suggested as an optimum in Ref. 6 would be much too high to control the Si layer thickness for ultrathin Si layers. We therefore lowered the temperature further. Best results with respect to an atomically smooth SOI film and a still intact BOX film were obtained for a substrate temperature of $750{ }^{\circ} \mathrm{C}$ with a silicon flux of $0.15 \pm 0.05 \mathrm{ML} / \mathrm{min}(0.003 \AA / \mathrm{s})$. The surface morphology obtained after evaporation for $30 \mathrm{~min}$ is shown in Fig. 1 by corresponding LEED and AFM images. LEED shows the expected $(2 \times 1)$ reconstructed pattern of a clean

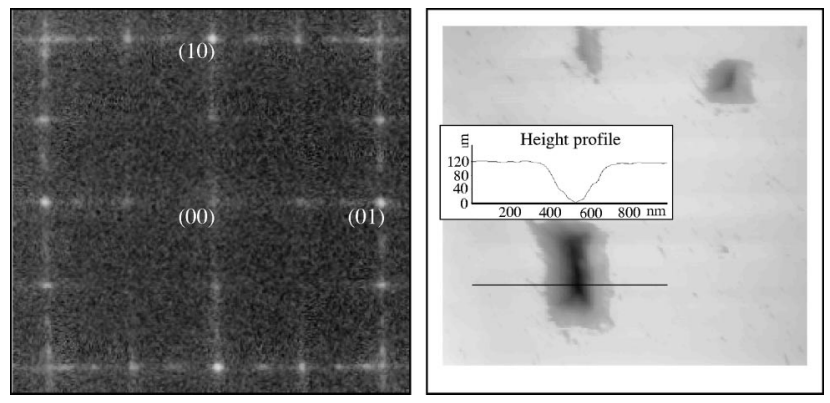

FIG. 2. Left: LEED pattern a SOI surface cleaned by Si evaporation at $T$ $=750{ }^{\circ} \mathrm{C}$ taken at an electron energy of $111 \mathrm{eV}$. Evaporation rate reduced to one half of that of Fig. 1. Right: AFM picture $\left(1.7 \times 1.7 \mu \mathrm{m}^{2}\right)$ of a SOI surface with pits after sputtering/oxygen treatment. The depth is around 100 $\mathrm{nm}$ as can be seen in the line profile.

$\mathrm{Si}(100)$ surface. From a quantitative LEED spot profile analysis at several scattering conditions we found an average terrace length of perfect (100)-oriented terraces larger than $400 \AA$. In addition, the vertical rms roughness, $\Delta$, was determined to be around $1.5 \AA$, as concluded from a $G(S)$-analysis of the specular beam. ${ }^{10}$ As seen from the right part of Fig. 1, AFM clearly shows a smooth and most notably continuous film of silicon. The native oxide layers have been removed completely, since AES shows only Si specific emission lines. The average rms roughness obtained by AFM (calculated from a $3.3 \times 3.3 \mu \mathrm{m}^{2}$ scan area) is $2.1 \AA$, in good agreement with LEED. After this treatment, the BOX layer turned out to be still intact, i.e., the resistance between Si layer and bulk silicon was still unmeasurably large $(\geqslant 50 \mathrm{M} \Omega$ ).

Indeed, the evaporation rate used in Fig. 1 turned out to be close to the lowest possible at the temperature of $750{ }^{\circ} \mathrm{C}$. The result of the same experiment with the evaporation rate only lowered by a factor of 2 at the same temperature is shown in Fig. 2. The LEED pattern shows, apart from the typical integer and half order diffraction spots also satellite spots, which shift their position as a function of electron energy. Plotting the positions of the satellite spots as a function of energy shows that they originate from (113)-facets on the surface, which form the side walls of etch pits, as shown by the AFM image.

As an alternative method for obtaining smooth SOI surfaces, also a combination of sputtering with $\mathrm{Ar}$ ions and smoothing the surface by subsequent dosing of molecular oxygen in the same elevated temperature range has been tested. In this case the native oxide was removed first by cycles of sputtering the SOI crystal $\left(E_{\mathrm{Ar}^{+}}=600 \mathrm{eV}, I\right.$ $=0.5 \mu \mathrm{A})$ and annealing at $750{ }^{\circ} \mathrm{C}$ until no traces of oxygen were visible with AES. Right after the sputtering/annealing cycles only a broad $(1 \times 1)$ LEED structure with a typical half width [full width at half maximum (FWHM)] of $10 \%$ Brillouin Zone of the specular beam at an out-of-phase condition was observed. Nevertheless, heating the surface up to $750^{\circ} \mathrm{C}$ in an $\mathrm{O}_{2}$ atmosphere of $2 \times 10^{-6} \mathrm{~Pa}$ results also in a high quality $\mathrm{Si}(100)$ surface almost comparable to that shown in Fig. 1. However, pit formation with (113) orientation as a consequence of the smoothing effect by preferential oxidation and desorption of $\mathrm{Si}$ atoms at step sites cannot be avoided, as tested with AFM.

The experiments described earlier show how a Si surplus at the surface can be used to remove oxide layers from SOI 


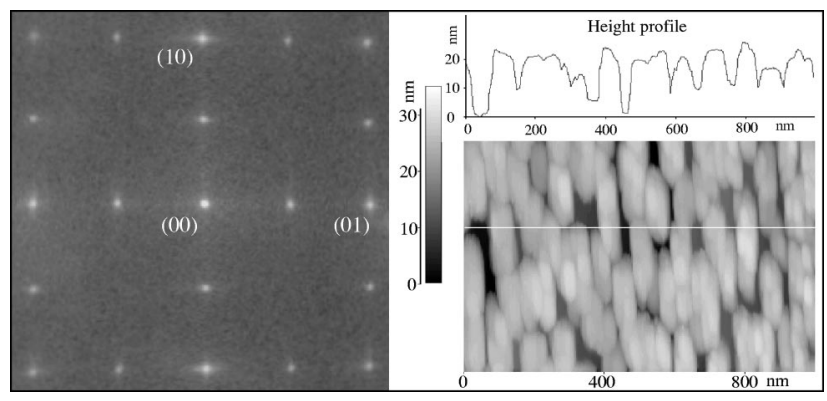

FIG. 3. LEED pattern (left, electron energy $82 \mathrm{eV}$ ) and AFM image (right) with a representative line scan of a SOI surface annealed to $1030^{\circ} \mathrm{C}$. The electron energy of $82 \mathrm{eV}$ corresponds to an in-phase scattering condition.

surfaces leaving atomically smooth $\mathrm{Si}$ surfaces. The same reaction, however, must also take place at the $\mathrm{Si} / \mathrm{SiO}_{2}$ interface, where the bulk Si material provides a huge reservoir of $\mathrm{Si}$ atoms. Nevertheless, it seems that SOI in some cases can stand at least temperatures up to $1000{ }^{\circ} \mathrm{C} .{ }^{1}$ Even more, flash cycles have been used to remove the native oxide like for silicon. The findings on our samples are clearly at variance with these statements.

After heating SOI samples, that have first been carefully degassed at $800^{\circ} \mathrm{C}$, to temperatures between 1000 and $1030^{\circ} \mathrm{C}$ for only $5 \mathrm{~s}$ at pressures below $10^{-7} \mathrm{~Pa}$, LEED sees a brilliant $(2 \times 1)$ structure [see Fig. 3(a)] with both reconstruction domains, indicating complete removal of the surface oxide layer and a perfect SOI film, in agreement with Ref. 1. The average terrace length of (100)-oriented terraces is around $260 \AA$, as determined from the FWHM of the (00)spot at an out-of-phase condition. Nevertheless, streaks around the diffraction spots are visible, indicating already a nonuniform surface. The finite resistance measured after this treatment between bulk and surface Si layer, however, shows that the BOX layer is obviously destroyed.

Ex situ AFM in contact mode shows that clusters $200 \mathrm{~nm}$ long and $40 \mathrm{~nm}$ wide have been formed (see right part of Fig. 3 ), most likely silicon clusters, that are consistent both with the measured terrace widths in LEED and with AES results. The finite size of the silicon clusters can be seen in the LEED pattern by the streaks around all diffraction spots. The elongated structure of the $\mathrm{Si}$ islands is most likely an elec- tromigration effect, a consequence of heating the surface with direct current.

These experiments show that also at the inner $\mathrm{Si} / \mathrm{SiO}_{2}$ interface the formation of volatile $\mathrm{SiO}$ is a fast process at temperatures around $1000^{\circ} \mathrm{C}$, leading to complete destruction of the SOI structure. However, even at $900 \mathrm{~K}$ this process was observed. At this temperature only a broad $(1 \times 1)$ structure was found after several minutes of annealing that could not be improved by further annealing steps, indicating only a poor crystalline quality, most likely because of intermixing of $\mathrm{Si}$ and nonstoichiometric oxide species. More important, the resistance across the buried oxide film, measured at room temperature, decreased significantly already after the first few minutes of annealing from $>50$ to $0.6 \mathrm{M} \Omega$, indicating the onset of the destructive etching process. In contrast, annealing at a temperature of $800^{\circ} \mathrm{C}$ over many hours caused no measurable changes. Even after $15 \mathrm{~h}$ of heating the sample the resistance measured between the bulk $\mathrm{Si}$ and the SOI film was unmeasurably large ( $\geqslant 50 \mathrm{M} \Omega$ ), i.e., temperatures of $800^{\circ} \mathrm{C}$ should not be exceeded in long time annealing steps. Similiar etching processes below $1000^{\circ} \mathrm{C}$ have been observed also by Ishikawa et $a .^{4}$ Interestingly, they used UNIBOND ${ }^{\circledR}$ SOI material, too.

The authors would like to thank Professor Dr. Latychev for many fruitful discussions during his stay in Hannover.

${ }^{1}$ K. C. Lin, O. W. Holland, L. C. Feldman, and H. H. Weitering, Appl. Phys. Lett. 72, 2313 (1998).

${ }^{2}$ M. Liehr, J. E. Lewis, and G. W. Rubloff, J. Vac. Sci. Technol. A 5, 1559 (1987).

${ }^{3}$ K. E. Johnson and T. Engel, Phys. Rev. Lett. 69, 339 (1992).

${ }^{4}$ Y. Ishikawa, M. Kumezawa, R. Nuryadi, and M. Tabe, Appl. Surf. Sci. 190, 11 (2002).

${ }^{5}$ Y.-K. Sun, D. J. Bonser, and T. Engel, J. Vac. Sci. Technol. A 10, 2314 (1992).

${ }^{6}$ G. D. Wilk, Y. Wei, H. Edwards, and R. M. Wallace, Appl. Phys. Lett. 70, 2288 (1997).

${ }^{7}$ A. A. Shklyaev, M. Shibata, and M. Ichikawa, Phys. Rev. B 62, 1540 (2000).

${ }^{8}$ T. Engel, Surf. Sci. Rep. 18, 91 (1993).

${ }^{9}$ C. A. Lent and P. J. Cohen, Surf. Sci. 139, 121 (1984).

${ }^{10}$ R. Tromp, G. W. Rubloff, P. Balk, F. K. LeGoues, and J. van Loenen, Phys. Rev. Lett. 55, 2332 (1985). 